\title{
Finite Element Analysis of a Lightweight Milling Cutter for Metal Additive Manufacturing
}

\author{
Pavel Hanzl ${ }^{1}$, Miroslav Zetek ${ }^{1}$, Vojtěch Rulc ${ }^{2}$, Hynek Purš², Ivana Zetková ${ }^{1}$ \\ ${ }^{1}$ Faculty of Mechanical Engineering, University of West Bohemia. Univerzitní 8, 30614 Pilsen. Czech Republic. E-mail: \\ hanzlp@rti.zcu.cz, mzetek@rti.zcu.cz, zetkova@rti.zcu.cz \\ ${ }^{2}$ Advanced Engineering s.r.o. Na Ostrohu 2405/16, 16000 Praha 6. Czech Republic. E-mail: vrulc@advanced-eng.cz, \\ info@advanced-eng.cz,
}

Previous work has proposed a process for implementing a lattice structure into a milling cutter body based on clustering in the milling cutter with modified main dimensions of a BCC cubic lattice structure cell. A finite element analysis model has been created to predict the strain and deformation in the struts of the lattice. The prediction made according to static loads demonstrates that the concept of a lightweight cutter meets the strength requirements, though its stiffness does not reach the fully-filled version. The methodologies for creating the FE model are described in this paper. HyperWorks with OptiStruct were used for these analyses. Local stiffness could be improved by varying the strut diameter or using a different type of basic cell for the lattice structure in problematic locations, especially in the area of the connection between the shell of the cutter and the lattice structures.

\section{Keywords: Milling Tool, Topological Optimization, Direct Melting Laser Sintering, Metal Additive Manufacturing}

\section{Introduction}

The development of cutting tools is driven by the need for productivity and efficiency. Continuous improvement is essential, and computer-aided simulations are very beneficial in this respect. One example is given in a paper by Tikal et al. [1] which deals with the application of FE modelling of machining. In general, simulation is a valuable aid when designing machine components. [2]

Recently, increased productivity of milling has been achieved by using Metal Additive Manufacturing (MAM) which is also known as $3 \mathrm{D}$ printing from metal. This technology allows great freedom in geometry design. In one case, a minimum 50\% improvement of performance was achieved by reducing the mass of the cutter. [9] In another case, the use of MAM allowed more inserts to be fitted in the cutter. [10] However, the topic of this paper is unique in regard to its new approach to the production and design of a milling cutter.

The process of reducing part weight while respecting strength requirements is called Topological Optimization (TO). One TO principle, implementation of porous structures, was used to create a lightweight milling cutter. There are many types of porous structures or metallic cellular materials, such as metal foams, truss structures, honeycombs and lattice structures produced by Metal Additive Manufacturing (MAM). [3]

Metal foams have a very irregular geometry which leads to localised damage at areas of intrinsic weakness under load. It has been shown that the cell walls of foams collapse by bending, rather than the more desirable stretching-dominated mode of deformation. [4] Research has proved that it is possible to design lightweight porous cores that offer better ratios of strength and stiffness to weight than those offered by metal foam materials [5] [8].

Design of porous cores should respect the possibilities of the production technology used. MAM technology is almost the only technology that is capable of producing a complex milling cutter with lattice structures from high strength steel, meaning that lattice structures can be used for TO. These lattice structures are regular and have an exact geometry. BCC topology was chosen which meets the requirements for manufacturability without support structures and appears to be very capable in terms of the orientation of its struts with respect to the force load vector. The issue of selection is described in more detail in a separate paper [6].

However, finding a suitable topology is always done on the principle of trial and error. [7] Significant selection efficiency in this case is achieved by deploying Finite Element Analysis. A comprehensive solution for topological optimization by lattice structures is a software solution such as OptiStruct. This structural solver together with a HyperMesh pre-processor is used for verification of the cutter design. Values of stress and material displacement are basic evaluation parameters which are complemented by the value of natural frequencies. The stress conditions and displacement values of the lightweight cutter were compared with the results of the fullsolid version.

Lightweight and energy-absorbing materials are interesting for a variety of industries, and the machining industry is no exception. The concept of a lightweight cutter has the potential to be successful in terms of endurance against cutting forces, and it turns out that even its natural frequencies are not so different.

\section{Model design}

A milling cutter is a rotating part and lattice structures are cubic. Attempts to bend the cubic structure to a radius results in a change in the topology geometry and disintegration in the strut nodes. This leads to a deterioration of load capacity. A better solution with respect to the cubic character of the lattice structure is an arrangement into clusters.

This solves the issue of how to implement the lattice structures with a cubic character into the rotating body of the cutter. A segment of cluster distributes the load from one insert. The angle segment is based on the number of inserts. Since the cutter has 6 inserts, each segment has $60^{\circ}\left(=360^{\circ} / 6\right)$. A circle split into segments according to this scheme is shown in Figure 1a. 


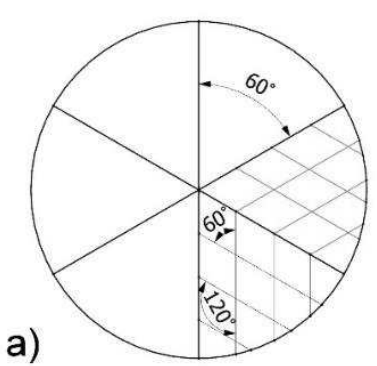

b)

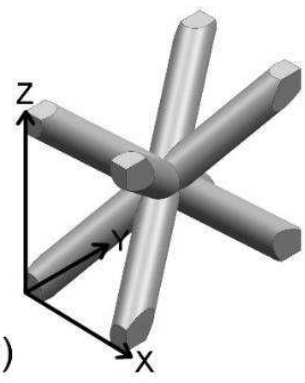

Fig. 1 a) Schematic view of six clusters and distributions of struts in two clusters b) Modified aspect ratio of the main length of the BCC cell sides

The BCC topology had to be modified to ensure that the struts across the cluster are linked continuously. The 2D scheme of strut distribution into one cluster is shown in Figure 1a. The original cubic BCC topology was changed according to this distribution. The aspect ratio of the basic BCC cell was changed from cubic to cuboid. The occupied volume is $6.3 \mathrm{~mm} \times 4 \mathrm{~mm} \times 7 \mathrm{~mm}$ ( $\mathrm{w} \mathrm{x} \mathrm{d} \mathrm{x}$ h). The 3D geometry of the basic cell is shown in Figure 1 b. The volume fraction was set at $18.7 \%$ and strut diameter is $1.19 \mathrm{~mm}$. Lattice structures in this cutter have a constant strut diameter. The struts grow at $38^{\circ}$, which is sufficient for the reliable production of BCC topology.

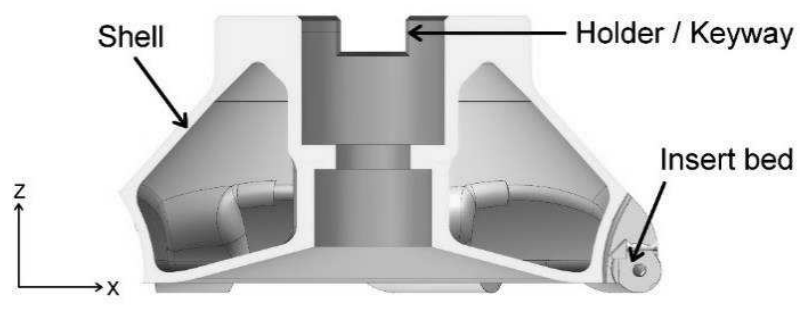

Fig. 2 Geometry of shell of milling cutter

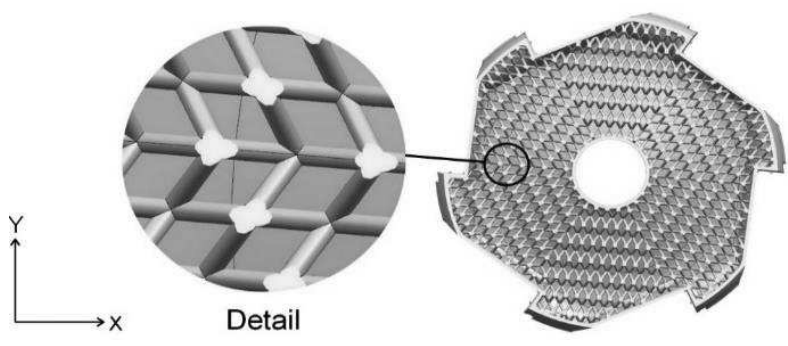

Fig. 3 Cluster distribution in circular field of lightweight cutter; detail with the connection method of the clusters

The prepared shell of the cutter body has a thickness of $2.3 \mathrm{~mm}$. The area of the inserts and keyway was strengthened to achieve maximum rigidity. The borders of the clusters were positioned before the inserts to ensure maximum absorption of shock generated during machining. The shell of the cutter is designed to be self-supporting. This means that its surfaces to the plane of the building platform of the MAM device have an angle higher than $40^{\circ}$. The outer diameter of the shell is $125 \mathrm{~mm}$ and its height is $62 \mathrm{~mm}$. The shell is shown in Figure 2. This regular lattice structure was inserted into the shell of the cutter. The resulting solution of the strut connection between the clusters is shown in detail in Figure 3.

\section{Finite Element Analysis}

The entire design of the cutter will be made from tool steel 1.2709 (European classification) and the machine EOS M 290 will be used for production. The mechanical properties for this material are the modulus of elasticity (210 GPa), Poisson ratio (0.3) and the density (7850 $\left.\mathrm{kg} \cdot \mathrm{m}^{-3}\right)$.

The boundary conditions such as force components were set based on experimental measurements of a solid cutter with the same geometry. Two inserts from six were always under full load during the simulation.

The value of the safety factor $(\mathrm{SF}=2)$ was chosen based on a rule of thumb to compensate for the difference between the dynamic character of a real load on the milling cutter and the static load of the simplistic model which is analysed. On the other hand, it is important to say that dynamic effects are covered just by the mentioned measurement and SF, so the simplification by converting the case into a static one should be fully adequate. After this, the force components were set as follows: $F_{c}=980 \mathrm{~N}, F_{f}=$ $550 \mathrm{~N}$ and $F_{p}=660 \mathrm{~N}$ (see Figure 4, image modified from Sandvik).
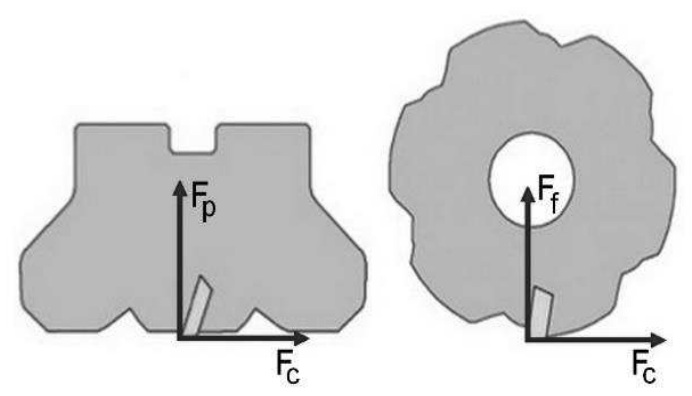

Fig. 4 Force component acting on the cutter body

Static stress and displacement analyses were carried out by solver OptiStruct in HyperWorks and the model was prepared with a HyperMesh pre-processor. The model is made up of 3D TETRA4 elements, which are tetrahedral. These elements are used for the whole volume except the lattice structure. For the lattice part, 1D CBEAM elements are used. The meshed model is shown in Figure 5a.

TIE contacts are modelled on the boundaries between the components to ensure shear and normal forces. Likewise, the relative position of contact surfaces is constrained. Attachment of the model on the spindle side is performed by the boundary conditions (Figure 6), which simulate a rotary symmetrical task. In total, three load cases are considered. The difference is in the position of the force pairs in relation to the keyway (see Figure 5b). 
a)

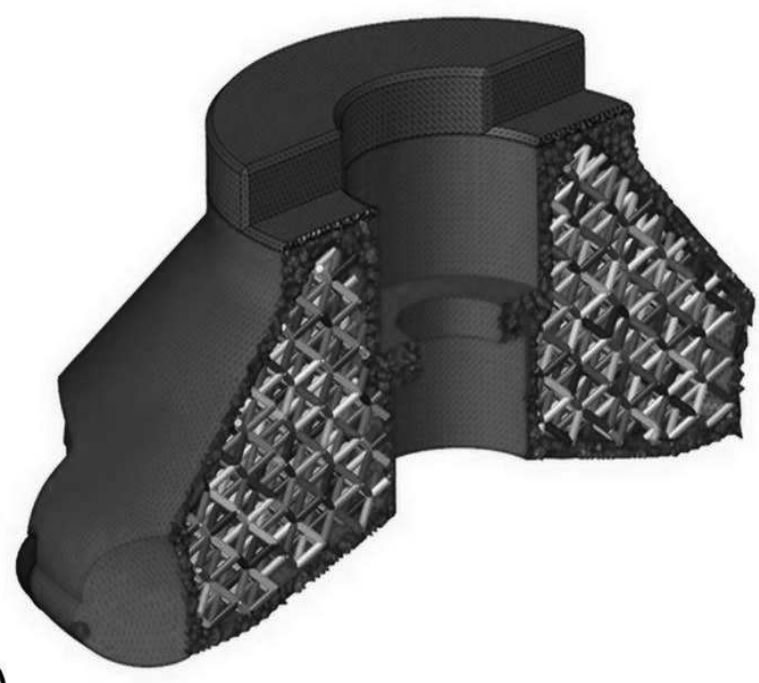

b)

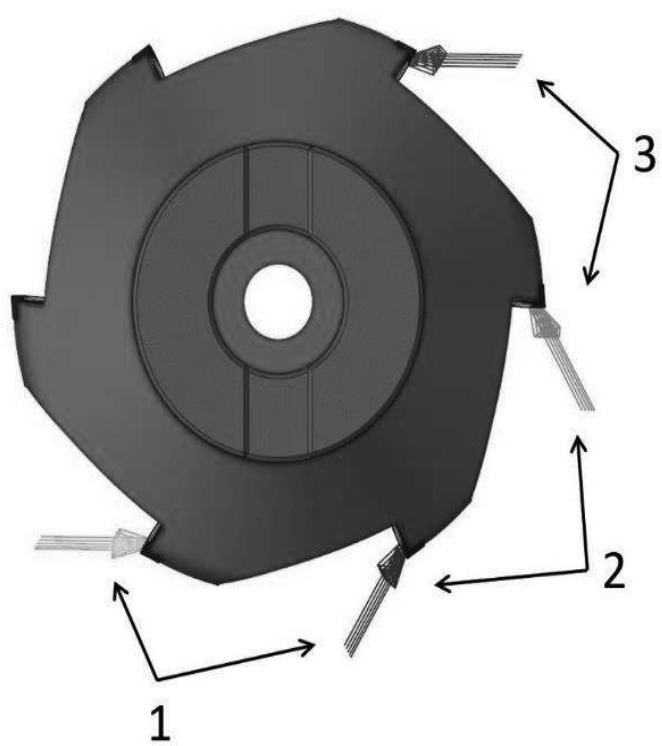

Fig. 5 a) Meshed model; b) Load cases of forces

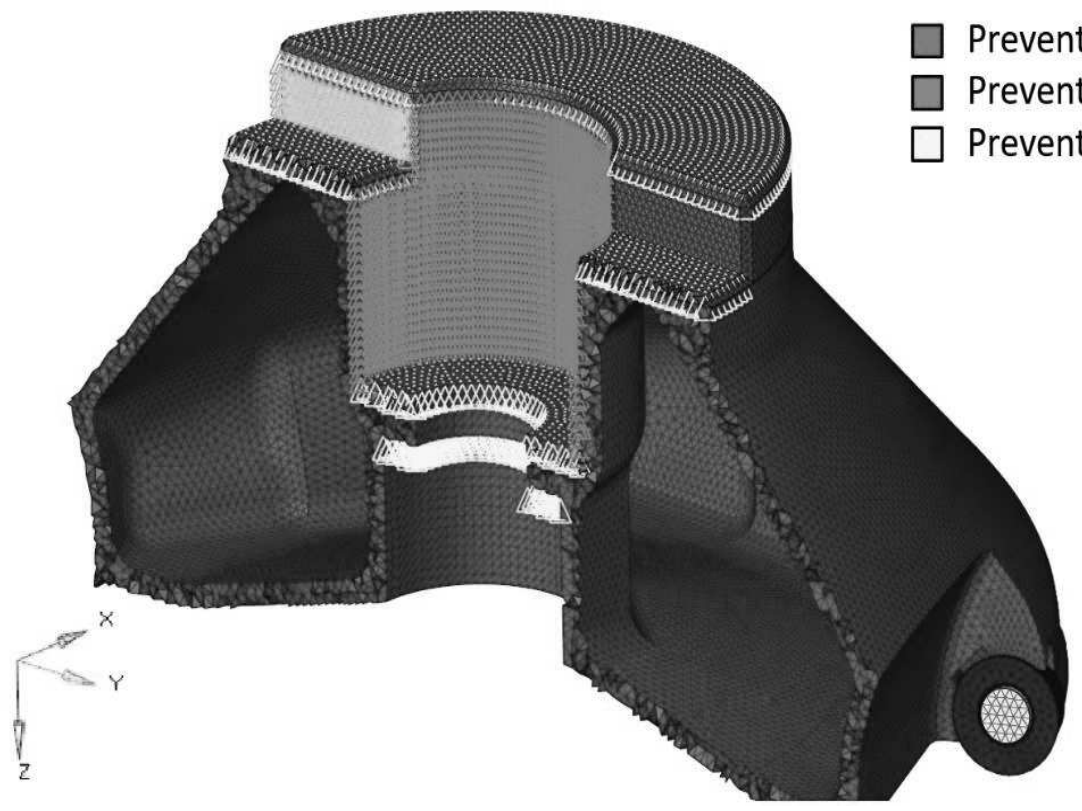

Fig. 6 TIE contacts of FE model; shell of lightweight cutter

\section{Results and discussion}

All three load cases for the lightweight cutter exhibit similar displacements and stresses. The analyses found that the maximum stress $(93.5 \mathrm{MPa})$ is reached in the area of the holder and the maximum value of the radial displacement of the insert centre was $1.99 \mu \mathrm{m}$. The maxima for the solid cutter are $71.3 \mathrm{MPa}$ and $0.58 \mu \mathrm{m}$ respectively. The stress and displacement fields of the lightweight cutter are shown in Figure 7.

The next highest maximum stress (42.5 MPa) is on the edge of the insert bed. The maximum stress in the lattice structure in the first load case is $12.6 \mathrm{MPa}$, but stress could reach up to four times $(50.4 \mathrm{MPa})$ in the joints of struts. This is caused because the stress in the struts modelled as CBEAM elements is evaluated only in the body of these elements. But in the joints, where the cross-section dimension is very similar to the strut cross-section, forces act from all the joining struts. If all of these forces have the same size, the stress in the joint is approximately 4 times higher than the stress in the struts.

For our further research there is a possibility to use the scripting abilities of HyperWorks and create a script which will help with accurate post-processing, and which will calculate the stress for every joint specifically, according to its cross-section (it is invariable in our case), and forces acting from neighbouring struts.

For a reference solid milling cutter, all deformations and displacements have a similar character and values. The maximum stress is measured again in the area of the cutter holder. The stress reaches $36.5 \mathrm{MPa}$ on the edge of the insert bed. The displacements for the load cases are shown in Table 1. The displacement values are measured at the centre of the screws. 


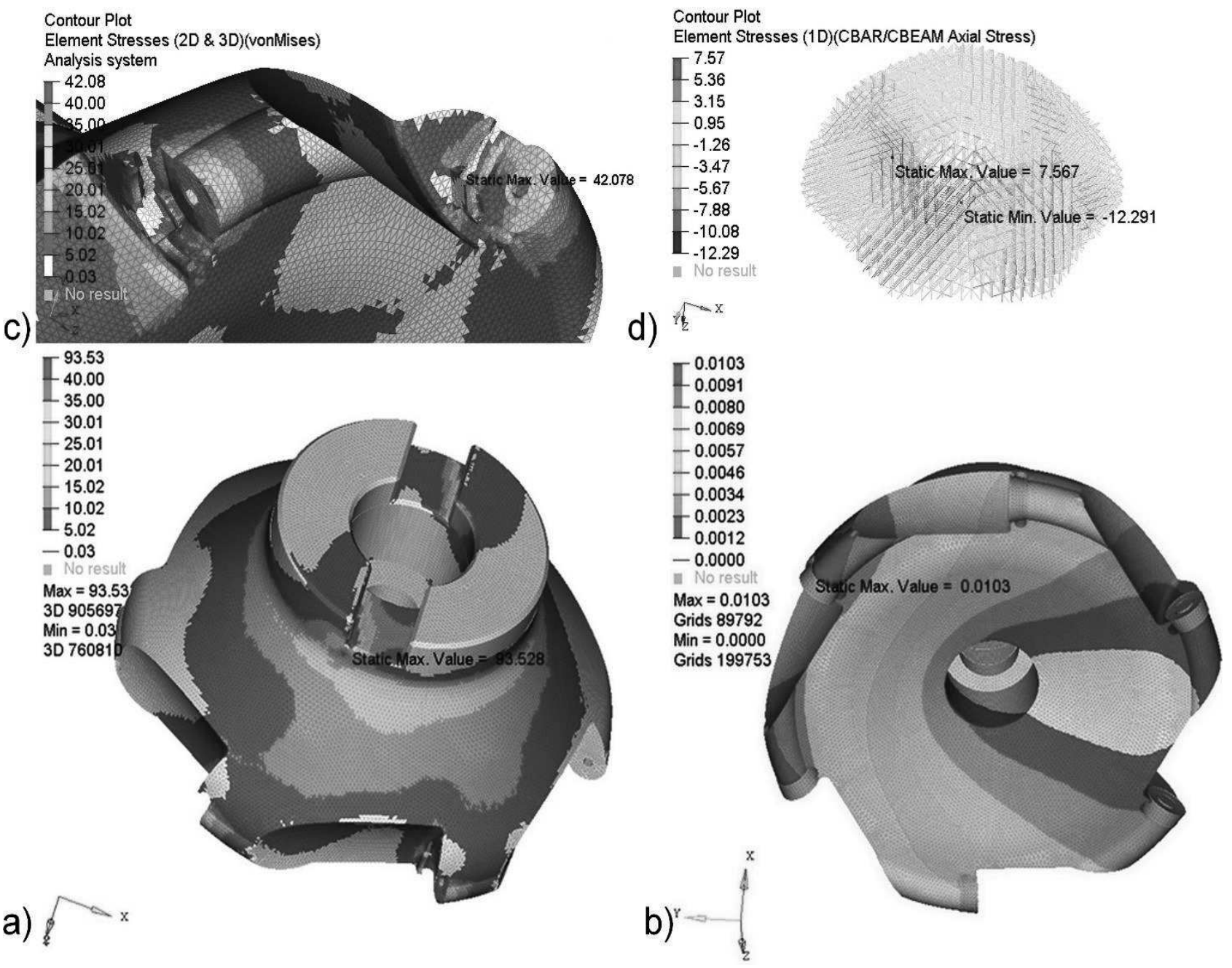

Fig. 7 Results of first load case; a) maximum stress in keyway; b) displacement analysis; c) detail of stress near insert bed; d) stress analysis of lattice structure

Tab. 1 Displacement [um] of insert during load case

\begin{tabular}{|c|c|c|c|c|c|c|c|c|c|}
\hline \multirow{2}{*}{ Model } & \multirow{2}{*}{$\begin{array}{l}\text { Load } \\
\text { case }\end{array}$} & \multicolumn{2}{|c|}{ Insert 1} & \multicolumn{2}{|c|}{ Insert 2} & \multicolumn{2}{|c|}{ Insert 3} & \multicolumn{2}{|c|}{ Insert 4} \\
\hline & & radial & $\operatorname{axis} \mathrm{z}$ & radial & axis $\mathrm{z}$ & radial & axis $\mathrm{z}$ & radial & $\operatorname{axis~} \mathrm{z}$ \\
\hline \multirow{3}{*}{ Lattice } & 1 & -0.958 & -0.270 & 1.891 & -3.585 & & & & \\
\hline & 2 & & & -0.937 & -0.440 & 1.986 & -3.326 & & \\
\hline & 3 & & & & & -0.878 & -0.162 & 1.970 & -3.419 \\
\hline \multirow{3}{*}{ Solid } & 1 & -0.007 & -0.378 & 0.575 & -1.098 & & & & \\
\hline & 2 & & & 0.071 & -0.553 & 0.521 & -0.899 & & \\
\hline & 3 & & & & & -0.011 & -0.319 & 0.514 & -0.925 \\
\hline
\end{tabular}

The next simulations focused on natural frequencies. A modal analysis of both milling cutters (lightweight and solid) was provided in solver OptiStruct again and the models were not fixed using boundary conditions. The measured values from the simulations are listed in Table 2. The first three natural shapes for frequencies match, but the other natural shapes of higher natural frequencies are different. Therefore, the first three values are important for comparison. These values are underlined in the table. Consequently, the differences in natural frequencies are on average $33 \%$ lower for the lightweight cutter in the range of $10.3-12.2 \mathrm{kHz}$.

Tab. 2 Natural frequencies [Hz]

\begin{tabular}{|l|l|l|l|l|l|l|l|l|l|l|}
\hline Model & $\mathbf{1}$ & $\mathbf{2}$ & $\mathbf{3}$ & $\mathbf{4}$ & $\mathbf{5}$ & $\mathbf{6}$ & $\mathbf{7}$ & $\mathbf{8}$ & $\mathbf{9}$ & $\mathbf{1 0}$ \\
\hline Lattice & $\underline{10295}$ & $\underline{11077}$ & $\underline{12176}$ & 14076 & 14106 & 14390 & 15863 & 16380 & 16432 & 17291 \\
\hline Solid & $\underline{15014}$ & $\underline{16391}$ & $\underline{18629}$ & 24036 & 24065 & 24165 & 26626 & 27134 & 27651 & 28556 \\
\hline
\end{tabular}




\section{Conclusion}

The main aim of this paper is to perform a static analysis in the linear region of the deformations. The subject of the analysis is a milling cutter that is filled with a regular lattice structure designed for a rotating cutter. The whole of this concept of a lightweight milling cutter combines the manufacturability using MAM and the static load requirements for machining by milling. A firm and lightweight lattice structure is crucial because it forms the core of the new mill design and affects the resulting behaviour during the cutting process.

However, lattice structures are not usually fully applicable for rotating designs due to their cubic character. This issue is solved by segmentation into clusters. The chosen BCC topology was modified in order to meet the requirement of this distribution into clusters.

The analyses were carried out and weaknesses were found in the cutter design. But the measured strength in the cutter and lattice structure was far from the value of the yield strength. The maximum stress $(93.5 \mathrm{MPa})$ was reached in the area of the holder. The maximum value of the radial displacement of the insert centre was $1.99 \mu \mathrm{m}$. In order to compare the results, the solid version of the cutter was also analysed. The maxima for the solid cutter were $71.3 \mathrm{MPa}$ for stress and $0.58 \mu \mathrm{m}$ for displacement. All deformations and displacements have a similar character to the lightweight version.

Direct comparison of both versions of the cutters revealed that the higher load in the area of the holder is caused by the lattice structure. The stress increase in the lattice variant is $31 \%$ higher than the full version. In the area of the inserts, the increase is $16 \%$. The results reveal that the solid variant is stiffer and shows less deformation than the lightweight variant.

The lower stiffness of the lightweight cutter is evident from the displacement for the first load case. The $\mathrm{z}$-axis deformation is lower for the lattice, but the deformation in the radial direction is orders of magnitude larger. However, the design of the lightweight cutter did not reveal any area which would compromise the coherence of the material during the expected load. The concept of dividing the lattice structure into clusters proved very effective. The strut connection between the clusters is rigid and shows no signs of overload.

The lower rigidity is confirmed by the natural frequencies. The differences in frequencies for the solid and the lightweight cutter is $33 \%$. The lower natural frequencies mean a less rigid body. However, the lower rigidity of the cutting tool does not necessarily mean worse performance, as verified by Miyaguchi et al. [11]. They found that the tool life of the mill end can be extended by reducing the rigidity of the cutting tool. This will be confirmed or refuted by real experiments focused on tool wear.

The maximum stress in the lattice structure in the first load case is $12.6 \mathrm{MPa}$, but the stress can reach up to four times this $(50.4 \mathrm{MPa})$ in the joints of the struts. In this case, the importance of the connection method is confirmed, because the peak stress is in this area. Further steps for improvement will be focused in this area. The SW OptiStruct may be helpful in this case as one of the great advantages of this SW is the possibility of a variable cross-section of the struts. Thus, a stronger connection between the shell and the porous structure can be achieved.

The stresses and displacement may be higher than in reality in the case of the lattice structure. Smith et al. [3] evaluated the conformity between the FEA results and the real behaviour of samples of the BCC structure. They found that the pressure samples have higher load capacity then the FEA results shown. The problem was allocated in the nodes of the lattice structure where extra metal material in the FE model had to be added for the same result.

Typical approaches to tool rigidity are based on additional weight which leads to a decrease in tool dynamics, which requires a more powerful spindle drive, which is more expensive and less energetically favourable. In this paper, a new advanced approach was presented, trying to reduce tool weight and increase efficiency using available MAM materials and design modifications by implementing a lattice structure. The results of the analyses confirmed that the new concept of the milling cutter is suitable for machining operations. This will be verified by experimental testing in the next stages. In total, the lightening of the milling cutter has the potential to bring a high degree of innovation.

Despande et al. [12] investigated the properties of an octet-truss lattice structure. It was shown that this topology is stretching-dominated and is preferred for hard applications. But its geometry has a higher number of struts per cell, which leads to smaller diameter struts than the BCC topology and problematic manufacturing when the volume fraction is the same for both topologies. However, the octet-truss lattice could ensure a better response of the lightweight milling cutter in the next step of improving the cutter properties.

The study by Smith et al. [3] proved that 3D continuum or 1D beam element types of FE modelling of lattice structures can be accurate. But some difficulties were identified. Modelling a large lattice structure becomes prohibitive and 1D beam modelling reduces the time taken for the analyses. Therefore this type of modelling was used in this paper. But there is a lack of contact at the nodal regions when using $1 \mathrm{D}$ beam elements. A solution could be to increase the strut diameter in these locations to compensate for this. [3]

The nominal/effective diameter of real struts within a lattice structure is difficult to estimate. This is the most serious problem for accurately measuring the material properties of a lattice structure. Reverse engineering could be used for this. Further work will address this topic.

\section{Acknowledgement}

This paper was created with the subsidy of the project TRIO FV30149.

\section{References}

[1] TIKAL, F., ZETEK, M., KAVALÍR, T. (2017) Application of FE Modelling of Machining Using 
DEFORM ${ }^{\mathrm{TM}}$, Published by Manufacturing Technology, ISSN 1213-2489, June 2017, Vol. 17, No. 3

[2] BLATNICKÝ, M., DIŽO, J., ŠTAUDEROVÁ, M. (2017) Strength Analysis of a Structure for Attachment of a Winch on SUV, Published by Manufacturing Technology, ISSN 1213-2489, June 2017, Vol. 17, No. 3

[3] SMITH, M., GUAN, Z., CANTWELL, W.J. (2013) Finite element modelling of the compressive response of lattice structures manufactured using the selective laser melting technique, International Journal of Mechanical Sciences, Vol. 67, Pp. 28-41, ISSN 0020-7403

[4] DESHPANDE, V.S., FLECK, N.A. (2001) Collapse of truss core sandwich beams in 3-point bending, International Journal of Solids and Structures, Vol. 38, Pp. 6275-6305

[5] DESHPANDE, V.S., ASHBY, M.F., FLECK, N.A. (2001) Foam topology: bending versus stretching dominated architectures, Acta Materialia, Vol. 49, Issue 6, Pp. 1035-1040, ISSN 1359-6454

[6] HANZL, P., ZETKOVÁ, I. (2019) Benefits of a new approach to designing milling cutter using Metal Additive Manufacturing, Published by Manufacturing Technology, ISSN 1213-2489, April 2019, Vol. 18, No. 2

[7] VERNON R.A. (2016) Discovering optimal unit cell configuration when designing for additive ma- nufacturing using lattice structures, Thesis, Department of Mechanical and Aerospace Engineering, California State University, Long Beach

[8] SYAM, W.P., JIANWEI, W., ZHAO, B., MASKERY, I., ELMADIH, W., LEACH, R. (2018) Design and analysis of strut-based lattice structures for vibration isolation, Published by Precision Engineering, ISSN 0141-6359, Vol. 52, Pp. 494-506.

[9] Design the unseen- optimize performance. Sandvik. [online]. [date 2019-06-05]. Available from: https://www.additive.sandvik/en/the-additive-advantage/design-the-unseen-optimize-performance/

[10] 3D-printed PCD milling cutters. KOMET JEL. [online]. [date 2019-06-07]. Available from: http://www.kometscandinavia.com/images/Komet_kataloger/1342CF11-9F85-4819-AD2A17AF4F0A7AF9webspeed.dk.pdf

[11] MIYAGUCHI, T., MASUDA, M., TAKEOKA, E., IWABE, H. (2001) Effect of tool stiffness upon wear in high spindle speed milling using small ball end mill, Precision Engineering, Volume 25, Issue 2, Pages 145-154, ISSN 0141-6359

[12] DESHPANDE, V.S., FLECK, N.A., ASHBY, M.F. (2001) Effective properties of the octet-truss lattice material, Journal of the Mechanics and Physics of Solids, Vol. 49, Issue 8, Pp. 1747-1769, ISSN 0022-5096 